PROCEEDINGS OF THE

AMERICAN MATHEMATICAL SOCIETY

Volume 128, Number 2, Pages 511-519

$\mathrm{S}$ 0002-9939(99)05261-2

Article electronically published on July 6, 1999

\title{
THE BEST POSSIBILITY OF THE GRAND FURUTA INEQUALITY
}

\author{
KÔTARÔ TANAHASHI
}

(Communicated by David R. Larson)

\begin{abstract}
Let $A, B \in B(H)$ be invertible bounded linear operators on a Hilbert space $H$ satisfying $O \leq B \leq A$, and let $p, r, s, t$ be real numbers satisfying $1<s, 0<t<1, t \leq r, 1 \leq p$. Furuta showed that if $0<\alpha \leq \frac{1-t+r}{(p-t) s+r}$, then $\left\{A^{\frac{r}{2}}\left(A^{-\frac{t}{2}} B^{p} A^{-\frac{t}{2}}\right)^{s} A^{\frac{r}{2}}\right\}^{\alpha} \leq A^{\{(p-t) s+r\} \alpha}$. This inequality is called the grand Furuta inequality, which interpolates the Furuta inequality $(t=0)$ and the Ando-Hiai inequality $(t=1, r=s)$.

In this paper, we show the grand Furuta inequality is best possible in the following sense: that is, if $\frac{1-t+r}{(p-t) s+r}<\alpha$, then there exist invertible matrices $A, B$ with $O \leq B \leq A$ which do not satisfy $\left\{A^{\frac{r}{2}}\left(A^{-\frac{t}{2}} B^{p} A^{-\frac{t}{2}}\right)^{s} A^{\frac{r}{2}}\right\}^{\alpha} \leq$ $A^{\{(p-t) s+r\} \alpha}$
\end{abstract}

\section{INTRODUCTION}

Let $A, B$ be bounded linear operators on a Hilbert space $H$. The following operator inequality is well-known as the Löwner-Heinz inequality ([9],[10]).

Proposition 1 ( Löwner-Heinz). Let $A, B \in B(H)$ be bounded linear operators on a Hilbert space $H$ satisfying $O \leq B \leq A$. If $0 \leq p \leq 1$, then $B^{p} \leq A^{p}$.

Concerning the Löwner-Heinz inequality, Chan and Kwong ([1]) conjectured that $O \leq B \leq A$ will imply $B^{2} \leq\left(B A^{2} B\right)^{\frac{1}{2}}$ and Furuta gave an affirmative answer, which is a very useful extension of the Löwner-Heinz inequality ([4]).

Proposition 2 (Furuta). Let $A, B \in B(H)$ be bounded linear operators on a Hilbert space $H$ satisfying $O \leq B \leq A$. If

$$
p+2 r \leq(1+2 r) q \text { and } 1 \leq q,
$$

then

$$
\left(A^{r} B^{p} A^{r}\right)^{\frac{1}{q}} \leq A^{\frac{p+2 r}{q}}
$$

and

$$
B^{\frac{p+2 r}{q}} \leq\left(B^{r} A^{p} B^{r}\right)^{\frac{1}{q}}
$$

Received by the editors September 27, 1997 and, in revised form, March 31, 1998.

1991 Mathematics Subject Classification. Primary 47B15.

Key words and phrases. The Löwner-Heinz inequality, the Furuta inequality, the grand Furuta inequality.

(C)1999 American Mathematical Society 


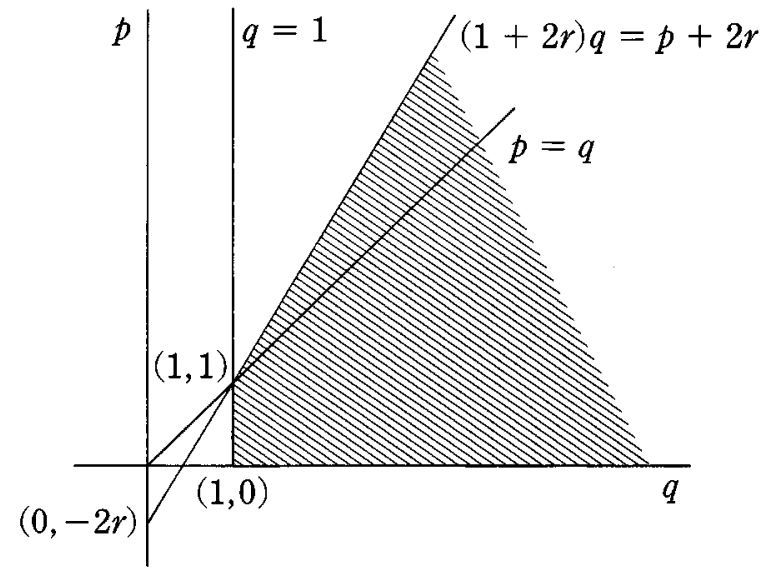

FiguRE 1

This inequality is called the Furuta inequality and many applications have been developed in the $p$-hyponormal operator theory ([2], [8]) and the relative entropy theory ([6], [7]). Recently, the Furuta inequality with negative powers has been studied by Yoshino ([13]), Fujii, Furuta, Kamei ([3]) and the author ([12]). Furthermore, Furuta ([7]) generalized the Furuta inequality.

Proposition 3 (Furuta). Let $A, B \in B(H)$ be invertible bounded linear operators on a Hilbert space $H$ satisfying $O \leq B \leq A$, and let $p, r, s, t$ be real numbers satisfying $1<s, 0<t<1, t \leq r, 1 \leq p$. If

$$
0<\alpha \leq \frac{1-t+r}{(p-t) s+r}
$$

then

$$
\left\{A^{\frac{r}{2}}\left(A^{-\frac{t}{2}} B^{p} A^{-\frac{t}{2}}\right)^{s} A^{\frac{r}{2}}\right\}^{\alpha} \leq A^{\{(p-t) s+r\} \alpha} .
$$

This inequality is called the grand Furuta inequality, which interpolates the Furuta inequality $(t=0)$ and the Ando-Hiai inequality $(t=1, r=s)$. In [12], the author showed that the condition (1.1) is best possible in the following sense: that is, if $0<q<1$ or $(1+2 r) q<p+2 r$, then there exist matrices $A, B$ with $O \leq B \leq A$ which do not satisfy the Furuta inequality (1.2). In this paper, by using the same technique as in [12], we show the condition (1.4) is best possible in the following sense: that is, if $\frac{1-t+r}{(p-t) s+r}<\alpha$, then there exist invertible matrices $A, B$ with $O \leq B \leq A$ which do not satisfy the grand Furuta inequality (1.5).

\section{RESUlT}

Theorem 4. Let $p, r, s, t$ be real numbers satisfying $1<s, 0<t<1, t \leq r, 1 \leq p$. If

$$
\frac{1-t+r}{(p-t) s+r}<\alpha
$$


then there exist invertible matrices $A, B$ with $O \leq B \leq A$ which do not satisfy

$$
\left\{A^{\frac{r}{2}}\left(A^{-\frac{t}{2}} B^{p} A^{-\frac{t}{2}}\right)^{s} A^{\frac{r}{2}}\right\}^{\alpha} \leq A^{\{(p-t) s+r\} \alpha} .
$$

Proof. Let

$$
A=\left(\begin{array}{cc}
a & \sqrt{\varepsilon(a-b-\delta)} \\
\sqrt{\varepsilon(a-b-\delta)} & b+\varepsilon+\delta
\end{array}\right)
$$

and

$$
B=\left(\begin{array}{ll}
1 & 0 \\
0 & b
\end{array}\right)
$$

where

$$
0<b<1<a, 0<\varepsilon, 0<\delta, \varepsilon(1-b) \leq \delta(a-1+\varepsilon) .
$$

Then $A$ and $B$ are invertible and $O \leq B \leq A$. We shall show that if (2.2) is valid, then we have a contradiction by letting

$$
\delta=\frac{1-b}{a-1} \varepsilon, \varepsilon \rightarrow+0, b \rightarrow+0 .
$$

Assume that (2.2) is valid. Let

$$
\gamma=a-b+\varepsilon-\delta
$$

and

$$
U=\frac{1}{\sqrt{\gamma}}\left(\begin{array}{cc}
\sqrt{a-b-\delta} & \sqrt{\varepsilon} \\
\sqrt{\varepsilon} & -\sqrt{a-b-\delta}
\end{array}\right) .
$$

Then $U$ is unitary and

$$
U^{*} A U=\left(\begin{array}{cc}
a+\varepsilon & 0 \\
0 & b+\delta
\end{array}\right) .
$$

Multiplying (2.2) by $U, U^{*}$, we have

$$
U^{*} A^{\{(p-t) s+r\} \alpha} U \geq\left\{U^{*} A^{\frac{r}{2}} U\left(U^{*} A^{-\frac{t}{2}} U U^{*} B^{p} U U^{*} A^{-\frac{t}{2}} U\right)^{s} U^{*} A^{\frac{r}{2}} U\right\}^{\alpha}
$$

and

$$
\begin{aligned}
& \left(\begin{array}{cc}
(a+\varepsilon)^{\{(p-t) s+r\} \alpha} & 0 \\
0 & (b+\delta)^{\{(p-t) s+r\} \alpha}
\end{array}\right) \\
& \geq\left[\begin{array}{cc}
(a+\varepsilon)^{\frac{r}{2}} & 0 \\
0 & (b+\delta)^{\frac{r}{2}}
\end{array}\right)\left\{\left(\begin{array}{cc}
(a+\varepsilon)^{-\frac{t}{2}} & 0 \\
0 & (b+\delta)^{-\frac{t}{2}}
\end{array}\right)\right. \\
& \left.\left.\times \quad U^{*}\left(\begin{array}{cc}
1 & 0 \\
0 & b^{p}
\end{array}\right) U\left(\begin{array}{cc}
(a+\varepsilon)^{-\frac{t}{2}} & 0 \\
0 & (b+\delta)^{-\frac{t}{2}}
\end{array}\right)\right\}^{s}\left(\begin{array}{cc}
(a+\varepsilon)^{\frac{r}{2}} & 0 \\
0 & (b+\delta)^{\frac{r}{2}}
\end{array}\right)\right]^{\alpha}
\end{aligned}
$$

Write

$$
\begin{aligned}
& \left(\begin{array}{cc}
(a+\varepsilon)^{-\frac{t}{2}} & 0 \\
0 & (b+\delta)^{-\frac{t}{2}}
\end{array}\right) U^{*}\left(\begin{array}{cc}
1 & 0 \\
0 & b^{p}
\end{array}\right) U\left(\begin{array}{cc}
(a+\varepsilon)^{-\frac{t}{2}} & 0 \\
0 & (b+\delta)^{-\frac{t}{2}}
\end{array}\right) \\
& \quad=\frac{1}{\gamma} D .
\end{aligned}
$$

Then

$$
D=\left(\begin{array}{ll}
A_{1} & A_{3} \\
A_{3} & A_{2}
\end{array}\right)
$$


where

$$
\begin{aligned}
& A_{1}=(a+\varepsilon)^{-t}\left(a-b-\delta+b^{p} \varepsilon\right) \\
& A_{2}=(b+\delta)^{-t}\left(\varepsilon+b^{p}(a-b-\delta)\right) \\
& A_{3}=(a+\varepsilon)^{-\frac{t}{2}}(b+\delta)^{-\frac{t}{2}}\left(1-b^{p}\right) \sqrt{\varepsilon} \sqrt{a-b-\delta}
\end{aligned}
$$

If $\varepsilon, \delta \rightarrow+0$, then

$$
A_{1} \rightarrow a^{-t}(a-b), A_{2} \rightarrow b^{p-t}(a-b) .
$$

Since we will consider the case $b \rightarrow+0, A_{1} \rightarrow a^{1-t}$ and $A_{2} \rightarrow 0$, we may assume

$$
0<A_{2}<A_{1} \text {. }
$$

Let

$$
V=\frac{1}{\sqrt{A_{1}-A_{2}+2 \varepsilon_{1}}}\left(\begin{array}{cc}
\sqrt{A_{1}-A_{2}+\varepsilon_{1}} & \sqrt{\varepsilon_{1}} \\
\sqrt{\varepsilon_{1}} & -\sqrt{A_{1}-A_{2}+\varepsilon_{1}}
\end{array}\right),
$$

where

$$
2 \varepsilon_{1}=-A_{1}+A_{2}+\sqrt{\left(A_{1}-A_{2}\right)^{2}+4 A_{3}^{2}} .
$$

Then $V$ is unitary and

$$
V^{*} D V=V^{*}\left(\begin{array}{ll}
A_{1} & A_{3} \\
A_{3} & A_{2}
\end{array}\right) V=\left(\begin{array}{cc}
A_{1}+\varepsilon_{1} & 0 \\
0 & A_{2}-\varepsilon_{1}
\end{array}\right) .
$$

Then (2.4) implies

$$
\begin{gathered}
\left(\begin{array}{cc}
(a+\varepsilon)^{\{(p-t) s+r\} \alpha} & 0 \\
0 & (b+\delta)^{\{(p-t) s+r\} \alpha}
\end{array}\right) \\
\geq\left\{\left(\begin{array}{cc}
(a+\varepsilon)^{\frac{r}{2}} & 0 \\
0 & (b+\delta)^{\frac{r}{2}}
\end{array}\right) V V^{*}\left(\frac{1}{\gamma} D\right)^{s} V V^{*}\left(\begin{array}{cc}
(a+\varepsilon)^{\frac{r}{2}} & 0 \\
0 & (b+\delta)^{\frac{r}{2}}
\end{array}\right)\right\}^{\alpha} \\
=\left\{\left(\begin{array}{cc}
(a+\varepsilon)^{\frac{r}{2}} & 0 \\
0 & (b+\delta)^{\frac{r}{2}}
\end{array}\right) V \frac{1}{\gamma^{s}}\left(\begin{array}{cc}
\left(A_{1}+\varepsilon_{1}\right)^{s} & 0 \\
0 & \left(A_{2}-\varepsilon_{1}\right)^{s}
\end{array}\right)\right. \\
\left.\times V^{*}\left(\begin{array}{cc}
(a+\varepsilon)^{\frac{r}{2}} & 0 \\
0 & (b+\delta)^{\frac{r}{2}}
\end{array}\right)\right\}^{\alpha} .
\end{gathered}
$$

Write the right-hand matrix as

$$
\gamma^{-s \alpha}\left(A_{1}-A_{2}+2 \varepsilon_{1}\right)^{-\alpha}\left(\begin{array}{cc}
B_{1} & B_{3} \\
B_{3} & B_{2}
\end{array}\right)^{\alpha}
$$

then

$$
\begin{aligned}
& B_{1}=(a+\varepsilon)^{r}\left\{\left(A_{1}+\varepsilon_{1}\right)^{s}\left(A_{1}-A_{2}+\varepsilon_{1}\right)+\left(A_{2}-\varepsilon_{1}\right)^{s} \varepsilon_{1}\right\}, \\
& B_{2}=(b+\delta)^{r}\left\{\left(A_{1}+\varepsilon_{1}\right)^{s} \varepsilon_{1}+\left(A_{2}-\varepsilon_{1}\right)^{s}\left(A_{1}-A_{2}+\varepsilon_{1}\right)\right\}, \\
& B_{3}=(a+\varepsilon)^{\frac{r}{2}}(b+\delta)^{\frac{r}{2}} \sqrt{\varepsilon_{1}} \sqrt{A_{1}-A_{2}+\varepsilon_{1}}\left\{\left(A_{1}+\varepsilon_{1}\right)^{s}-\left(A_{2}-\varepsilon_{1}\right)^{s}\right\} .
\end{aligned}
$$

If $\varepsilon, \delta \rightarrow+0$, then

$$
\varepsilon_{1} \rightarrow 0, B_{1} \rightarrow a^{r-s t}(a-b)^{1+s}\left(a^{-t}-b^{p-t}\right), B_{2} \rightarrow b^{r+(p-t) s}(a-b)^{1+s}\left(a^{-t}-b^{p-t}\right) .
$$

Since we will consider the case $b \rightarrow+0, B_{1} \rightarrow a^{1+r+s-t-s t}$ and $B_{2} \rightarrow 0$, we may assume

$$
0<B_{2}<B_{1} \text {. }
$$


Let

$$
W=\frac{1}{\sqrt{B_{1}-B_{2}+2 \varepsilon_{2}}}\left(\begin{array}{cc}
\sqrt{B_{1}-B_{2}+\varepsilon_{2}} & \sqrt{\varepsilon_{2}} \\
\sqrt{\varepsilon_{2}} & -\sqrt{B_{1}-B_{2}+\varepsilon_{2}}
\end{array}\right)
$$

where

$$
2 \varepsilon_{2}=-B_{1}+B_{2}+\sqrt{\left(B_{1}-B_{2}\right)^{2}+4 B_{3}^{2}} .
$$

Then $W$ is unitary and

$$
W^{*}\left(\begin{array}{ll}
B_{1} & B_{3} \\
B_{3} & B_{2}
\end{array}\right) W=\left(\begin{array}{cc}
B_{1}+\varepsilon_{2} & 0 \\
0 & B_{2}-\varepsilon_{2}
\end{array}\right) .
$$

Multiplying (2.5) by $W^{*}, W$, we have

$$
\begin{gathered}
\gamma^{-s \alpha}\left(A_{1}-A_{2}+2 \varepsilon_{1}\right)^{-\alpha}\left(\begin{array}{cc}
\left(B_{1}+\varepsilon_{2}\right)^{\alpha} & 0 \\
0 & \left(B_{2}-\varepsilon_{2}\right)^{\alpha}
\end{array}\right) \\
\leq W^{*}\left(\begin{array}{cc}
(a+\varepsilon)^{\{(p-t) s+r\} \alpha} & 0 \\
0 & (b+\delta)^{\{(p-t) s+r\} \alpha}
\end{array}\right) W .
\end{gathered}
$$

Write the right-hand matrix as

$$
\frac{1}{B_{1}-B_{2}+2 \varepsilon_{2}}\left(\begin{array}{ll}
C_{1} & C_{3} \\
C_{3} & C_{2}
\end{array}\right)
$$

then

$$
\begin{aligned}
& C_{1}=(a+\varepsilon)^{\tilde{\alpha}}\left(B_{1}-B_{2}+\varepsilon_{2}\right)+(b+\delta)^{\tilde{\alpha}} \varepsilon_{2}, \\
& C_{2}=(a+\varepsilon)^{\tilde{\alpha}} \varepsilon_{2}+(b+\delta)^{\tilde{\alpha}}\left(B_{1}-B_{2}+\varepsilon_{2}\right), \\
& C_{3}=\left\{(a+\varepsilon)^{\tilde{\alpha}}-(b+\delta)^{\tilde{\alpha}}\right\} \sqrt{\varepsilon_{2}} \sqrt{B_{1}-B_{2}+\varepsilon_{2}}
\end{aligned}
$$

where

$$
\tilde{\alpha}=\alpha(r+s p-s t) .
$$

Let

$$
\tilde{\gamma}=\gamma^{s \alpha}\left(A_{1}-A_{2}+2 \varepsilon_{1}\right)^{\alpha}
$$

Then

$$
O \leq\left(\begin{array}{cc}
\tilde{\gamma} C_{1}-\left(B_{1}-B_{2}+2 \varepsilon_{2}\right)\left(B_{1}+\varepsilon_{2}\right)^{\alpha} & \tilde{\gamma} C_{3} \\
\tilde{\gamma} C_{3} & \tilde{\gamma} C_{2}-\left(B_{1}-B_{2}+2 \varepsilon_{2}\right)\left(B_{2}-\varepsilon_{2}\right)^{\alpha}
\end{array}\right) .
$$

Hence, by taking the determinant of the right-hand matrix, we have

$$
\begin{aligned}
0 \leq \tilde{\gamma}^{2} C_{1} C_{2}-\tilde{\gamma} C_{1}\left(B_{1}-B_{2}+2 \varepsilon_{2}\right)\left(B_{2}-\varepsilon_{2}\right)^{\alpha}-\tilde{\gamma} C_{2}\left(B_{1}-B_{2}+2 \varepsilon_{2}\right)\left(B_{1}+\varepsilon_{2}\right)^{\alpha} \\
+\left(B_{1}-B_{2}+2 \varepsilon_{2}\right)^{2}\left(B_{1}+\varepsilon_{2}\right)^{\alpha}\left(B_{2}-\varepsilon_{2}\right)^{\alpha}-\tilde{\gamma}^{2} C_{3}^{2},
\end{aligned}
$$

and

$$
\begin{aligned}
0 \leq\left(B_{1}-B_{2}+2 \varepsilon_{2}\right)\left\{\tilde{\gamma}^{2}(\alpha\right. & +\varepsilon)^{\tilde{\alpha}}(b+\varepsilon)^{\tilde{\alpha}}\left(B_{1}-B_{2}+2 \varepsilon_{2}\right) \\
& -\tilde{\gamma}(\alpha+\varepsilon)^{\tilde{\alpha}}\left(B_{1}-B_{2}+2 \varepsilon_{2}\right)\left(B_{2}-\varepsilon_{2}\right)^{\alpha} \\
& -\tilde{\gamma}(b+\varepsilon)^{\tilde{\alpha}}\left(B_{2}-\varepsilon_{2}\right)^{\alpha}-\tilde{\gamma}(\alpha+\varepsilon)^{\tilde{\alpha}} \varepsilon_{2}\left(B_{1}+\varepsilon_{2}\right)^{\alpha} \\
& -\tilde{\gamma}(b+\varepsilon)^{\tilde{\alpha}}\left(B_{1}-B_{2}+\varepsilon_{2}\right)\left(B_{1}+\varepsilon_{2}\right)^{\alpha} \\
& \left.+\left(B_{1}-B_{2}+2 \varepsilon_{2}\right)\left(B_{1}+\varepsilon_{2}\right)^{\alpha}\left(B_{2}-\varepsilon_{2}\right)^{\alpha}\right\}
\end{aligned}
$$


Since $0<B_{1}-B_{2}+2 \varepsilon_{2}$, we have

$$
\begin{aligned}
& -\varepsilon_{2}\left\{\tilde{\gamma}(a+\varepsilon)^{\tilde{\alpha}}-\left(B_{2}-\varepsilon_{2}\right)^{\alpha}\right\}\left\{\tilde{\gamma}(b+\delta)^{\tilde{\alpha}}-\left(B_{1}+\varepsilon_{2}\right)^{\alpha}\right\} \\
& \leq\left(B_{1}-B_{2}+\varepsilon_{2}\right)\left\{\tilde{\gamma}(a+\varepsilon)^{\tilde{\alpha}}-\left(B_{1}+\varepsilon_{2}\right)^{\alpha}\right\}\left\{\tilde{\gamma}(b+\delta)^{\tilde{\alpha}}-\left(B_{2}-\varepsilon_{2}\right)^{\alpha}\right\} .
\end{aligned}
$$

We estimate the first order of each terms in (2.7) with respect to $\varepsilon, \delta$. In the following, $o$ means $o(\varepsilon), o(\delta)$, that is

$$
\frac{o}{\varepsilon}, \frac{o}{\delta} \rightarrow 0 \quad(\varepsilon, \delta \rightarrow+0) .
$$

Then

$$
\begin{aligned}
A_{1} & =a^{-t}\left(1-\frac{t}{a} \varepsilon+o\right)(a-b)\left(1+\frac{b^{p}}{a-b} \varepsilon-\frac{1}{a-b} \delta+o\right) \\
& =a^{-t}(a-b)\left(1-\frac{t}{a} \varepsilon+\frac{b^{p}}{a-b} \varepsilon-\frac{1}{a-b} \delta+o\right), \\
A_{2} & =b^{-t}\left(1-\frac{t}{b} \delta+o\right) b^{p}(a-b)\left(1+\frac{1}{b^{p}(a-b)} \varepsilon-\frac{1}{a-b} \delta+o\right) \\
& =b^{p-t}(a-b)\left(1+\frac{1}{b^{p}(a-b)} \varepsilon-\frac{t}{b} \delta-\frac{1}{a-b} \delta+o\right), \\
A_{3}^{2} & =a^{-t}\left(1-\frac{t}{a} \varepsilon+o\right) b^{-t}\left(1-\frac{t}{b} \delta+o\right)\left(1-b^{p}\right)^{2} \varepsilon(a-b)\left(1-\frac{1}{a-b} \delta\right) \\
& =a^{-t} b^{-t}\left(1-b^{p}\right)(a-b) \varepsilon\left(1-\frac{t}{a} \varepsilon-\frac{t}{b} \delta-\frac{1}{a-b} \delta+o\right), \\
\varepsilon_{1} & =\frac{1}{2}\left(A_{1}-A_{2}\right)\left\{-1+\sqrt{1+\frac{4 A_{3}^{2}}{\left(A_{1}-A_{2}\right)^{2}}}\right\} \\
& =\frac{1}{2}\left(A_{1}-A_{2}\right)\left\{-1+\left(1+\frac{1}{2} \frac{4 A_{3}^{2}}{\left(A_{1}-A_{2}\right)^{2}}+o\right)\right\} \\
& =\frac{a^{-t} b^{-t}\left(1-b^{p}\right)^{2}}{a^{-t}-b^{p-t}} \varepsilon\left(1+\frac{o}{\varepsilon}\right) \cdot
\end{aligned}
$$

Hence

$$
\begin{aligned}
A_{1}-A_{2}+2 \varepsilon_{1} & =(a-b)\left(a^{-t}-b^{p-t}\right)\left(1+c_{1} \varepsilon+c_{2} \delta+o\right), \\
A_{1}-A_{2}+\varepsilon_{1} & =(a-b)\left(a^{-t}-b^{p-t}\right)\left(1+c_{3} \varepsilon+c_{4} \delta+o\right),
\end{aligned}
$$

and

$$
\tilde{\gamma}=(a-b)^{\alpha+\alpha s}\left(a^{-t}-b^{p-t}\right)^{\alpha}\left(1+\frac{\alpha s}{a-b} \varepsilon+\alpha c_{1} \varepsilon-\frac{\alpha s}{a-b} \delta+\alpha c_{2} \delta+o\right),
$$

where

$$
\begin{aligned}
& c_{1}=\frac{1}{(a-b)\left(a^{-t}-b^{p-t}\right)}\left(-t(a-b) a^{-t-1}+a^{-t} b^{p}-b^{-t}+\frac{2 a^{-t} b^{-t}\left(1-b^{p}\right)^{2}}{a^{-t}-b^{p-t}}\right), \\
& c_{2}=\frac{1}{(a-b)\left(a^{-t}-b^{p-t}\right)}\left(-a^{-t}+t(a-b) b^{p-t-1}+b^{p-t}\right), \\
& c_{3}=c_{1}-\frac{a^{-t} b^{-t}\left(1-b^{p}\right)^{2}}{(a-b)\left(a^{-t}-b^{p-t}\right)^{2}}, \\
& c_{4}=c_{2} .
\end{aligned}
$$


Then

$$
\begin{aligned}
& \left(A_{1}+\varepsilon_{1}\right)^{s} \\
& =a^{-s t}(a-b)^{s}\left(1-\frac{s t}{a} \varepsilon+\frac{s b^{p}}{a-b} \varepsilon+\frac{s b^{-t}\left(1-b^{p}\right)^{2}}{(a-b)\left(a^{-t}-b^{p-t}\right)} \varepsilon-\frac{s}{a-b} \delta+o\right)
\end{aligned}
$$

and

$$
\begin{aligned}
& \left(A_{2}-\varepsilon_{1}\right)^{s} \\
& \quad=b^{s p-s t}(a-b)^{s}\left(1+\frac{s}{b^{p}(a-b)} \varepsilon-\frac{s a^{-t}\left(1-b^{p}\right)^{2}}{b^{p}(a-b)\left(a^{-t}-b^{p-t}\right)} \varepsilon-\frac{s t}{b} \delta-\frac{s}{a-b} \delta+o\right) .
\end{aligned}
$$

Hence

$$
\begin{aligned}
B_{1} & =(a+\varepsilon)^{r}\left\{\left(A_{1}+\varepsilon_{1}\right)^{s}\left(A_{1}-A_{2}+\varepsilon_{1}\right)+\left(A_{2}-\varepsilon_{1}\right)^{s} \varepsilon_{1}\right\} \\
& =a^{r-s t}(a-b)^{1+s}\left(a^{-t}-b^{p-t}\right)\left(1+c_{5} \varepsilon+c_{6} \delta+o\right), \\
B_{2} & =(b+\delta)^{r}\left\{\left(A_{1}+\varepsilon_{1}\right)^{s} \varepsilon_{1}+\left(A_{2}-\varepsilon_{1}\right)^{s}\left(A_{1}-A_{2}+\varepsilon_{1}\right)\right\} \\
& =b^{r+s p-s t}(a-b)^{1+s}\left(a^{-t}-b^{p-t}\right)\left(1+c_{7} \varepsilon+c_{8} \delta+o\right), \\
B_{3}^{2} & =(a+\varepsilon)^{r}(b+\delta)^{r} \varepsilon_{1}\left(A_{1}-A_{2}+\varepsilon_{1}\right)\left\{\left(A_{1}+\varepsilon_{1}\right)^{s}-\left(A_{2}-\varepsilon_{1}\right)^{s}\right\}^{2} \\
& =a^{r-t} b^{r-t}(a-b)^{1+2 s}\left(a^{-s t}-b^{s p-s t}\right)^{2}\left(1-b^{p}\right)^{2} \varepsilon\left(1+\frac{o}{\varepsilon}\right), \\
\varepsilon_{2} & =\frac{1}{2}\left(B_{1}-B_{2}\right)\left\{-1+\sqrt{1+\frac{4 B_{3}^{2}}{\left(B_{1}-B_{2}\right)^{2}}}\right\} \\
& =\frac{1}{2}\left(B_{1}-B_{2}\right)\left\{-1+\left(1+\frac{1}{2} \frac{4 B_{3}^{2}}{\left(B_{1}-B_{2}\right)^{2}}+o\right)\right\} \\
& =\frac{a^{r-t} b^{r-t}(a-b)^{s}\left(a^{-s t}-b^{s p-s t}\right)^{2}\left(1-b^{p}\right)^{2}}{\left(a^{-t}-b^{p-t}\right)\left(a^{r-s t}-b^{r+s p-s t}\right)} \varepsilon\left(1+\frac{o}{\varepsilon}\right),
\end{aligned}
$$

where

$$
\begin{aligned}
& c_{5}=c_{3}+\frac{r-s t}{a}+\frac{s b^{p}}{a-b}+\frac{s b^{-t}\left(1-b^{p}\right)^{2}}{(a-b)\left(a^{-t}-b^{p-t}\right)}+\frac{a^{-t+s t} b^{-t+s p-s t}\left(1-b^{p}\right)^{2}}{(a-b)\left(a^{-t}-b^{p-t}\right)^{2}}, \\
& c_{6}=c_{4}-\frac{s}{a-b}, \\
& c_{7}=c_{3}+\frac{s}{b^{p}(a-b)}+\frac{a^{-t-s t} b^{-t-s p+s t}\left(1-b^{p}\right)^{2}}{(a-b)\left(a^{-t}-b^{p-t}\right)^{2}}-\frac{s a^{-t}\left(1-b^{p}\right)^{2}}{b^{p}(a-b)\left(a^{-t}-b^{p-t}\right)}, \\
& c_{8}=c_{4}+\frac{r-s t}{b}-\frac{s}{a-b} .
\end{aligned}
$$

Then

$$
\begin{aligned}
\tilde{\gamma}(b+\delta)^{\tilde{\alpha}}= & \gamma^{\alpha s}\left(A_{1}-A_{2}+2 \varepsilon_{1}\right)^{\alpha}(b+\delta)^{\alpha(r+s p-s t)} \\
= & b^{\alpha(r+s p-s t)}(a-b)^{\alpha(1+s)}\left(a^{-t}-b^{p-t}\right)^{\alpha} \\
& \times\left(1+\alpha c_{1} \varepsilon+\frac{\alpha s}{a-b} \varepsilon+\alpha c_{2} \delta-\frac{\alpha s}{a-b} \delta+\frac{\alpha(r+s p-s t)}{b} \delta+o\right), \\
\left(B_{1}+\varepsilon_{2}\right)^{\alpha}= & a^{\alpha(r-s t)}(a-b)^{\alpha(1+s)}\left(a^{-t}-b^{p-t}\right)^{\alpha}\left(1+\alpha c_{9} \varepsilon+\alpha c_{6} \delta+o\right), \\
\left(B_{2}-\varepsilon_{2}\right)^{\alpha}= & b^{\alpha(r+s p-s t)}(a-b)^{\alpha(1+s)}\left(a^{-t}-b^{p-t}\right)^{\alpha}\left(1+\alpha c_{10} \varepsilon+\alpha c_{8} \delta+o\right),
\end{aligned}
$$


where

$$
\begin{gathered}
c_{9}=c_{5}+\frac{a^{-t+s t} b^{r-t}\left(1-b^{p}\right)^{2}\left(a^{-s t}-b^{s p-s t}\right)^{2}}{(a-b)\left(a^{-t}-b^{p-t}\right)^{2}\left(a^{r-s t}-b^{r+s p-s t}\right)}, \\
c_{10}=c_{7}-\frac{a^{r-t} b^{-t-s p+s t}\left(1-b^{p}\right)^{2}\left(a^{-s t}-b^{s p-s t}\right)^{2}}{(a-b)\left(a^{-t}-b^{p-t}\right)^{2}\left(a^{r-s t}-b^{r+s p-s t}\right)} .
\end{gathered}
$$

Hence

$$
\begin{aligned}
& \tilde{\gamma}(b+\delta)^{\tilde{\alpha}}-\left(B_{2}-\varepsilon_{2}\right)^{\alpha} \\
& =b^{\alpha(r+s p-s t)}(a-b)^{\alpha(1+s)}\left(a^{-t}-b^{p-t}\right)^{\alpha} \\
& \quad \times\left(\alpha c_{1} \varepsilon+\frac{\alpha s}{a-b} \varepsilon+\alpha c_{2} \delta-\frac{\alpha s}{a-b} \delta+\frac{\alpha(r+s p-s t)}{b} \delta\right. \\
& \left.\quad-\alpha c_{10} \varepsilon-\alpha\left(c_{2}+\frac{r-s t}{b}-\frac{s}{a-b}\right) \delta+o\right) \\
& =b^{\alpha(r+s p-s t)}(a-b)^{\alpha(1+s)}\left(a^{-t}-b^{p-t}\right)^{\alpha} \varepsilon\left(c_{11}+\frac{\alpha s p}{b} \frac{\delta}{\varepsilon}+\frac{o}{\varepsilon}\right)
\end{aligned}
$$

where

$$
c_{11}=-\frac{\alpha s\left(1-b^{p}\right)\left(a^{-t}-b^{-t}\right)}{(a-b)\left(a^{-t}-b^{p-t}\right)}-\frac{\alpha a^{-t} b^{-t}\left(1-b^{p}\right)^{2}\left(a^{r}-b^{s}\right)\left(a^{-s t}-b^{s p-s t}\right)}{(a-b)\left(a^{-t}-b^{p-t}\right)^{2}\left(a^{r-s t}-b^{r+s p-s t}\right)} .
$$

Also, we have

$$
\begin{aligned}
& \tilde{\gamma}(a+\varepsilon)^{\tilde{\alpha}}-\left(B_{2}-\varepsilon_{2}\right)^{\alpha} \\
& \quad=\left(a^{\alpha(r+s p-s t)}-b^{\alpha(r+s p-s t)}\right)(a-b)^{\alpha(1+s)}\left(a^{-t}-b^{p-t}\right)^{\alpha}\left(1+\frac{o}{\varepsilon}\right), \\
& \tilde{\gamma}(b+\delta)^{\tilde{\alpha}}-\left(B_{1}+\varepsilon_{2}\right)^{\alpha} \\
& \quad=-\left(a^{\alpha(r-s t)}-b^{\alpha(r+s p-s t)}\right)(a-b)^{\alpha(1+s)}\left(a^{-t}-b^{p-t}\right)^{\alpha}\left(1+\frac{o}{\varepsilon}\right), \\
& B_{1}-B_{2}+\varepsilon_{2} \\
& \quad=(a-b)^{1+s}\left(a^{-t}-b^{p-t}\right)\left(a^{r-s t}-b^{r+s p-s t}\right)\left(1+\frac{o}{\varepsilon}\right), \\
& \tilde{\gamma}(a+\varepsilon)^{\tilde{\alpha}}-\left(B_{1}+\varepsilon_{2}\right)^{\alpha} \\
& \quad=a^{\alpha(r-s t)}\left(a^{\alpha s p}-1\right)(a-b)^{\alpha(1+s)}\left(a^{-t}-b^{p-t}\right)^{\alpha}\left(1+\frac{o}{\varepsilon}\right) .
\end{aligned}
$$

Hence, by (2.4),

$$
\begin{aligned}
& \left(a^{-s t}-b^{s p-s t}\right)^{2}\left(a^{\alpha(r+s p-s t)}-b^{\alpha(r+s p-s t)}\right)\left(a^{\alpha(r-s t)}-b^{\alpha(r+s p-s t)}\right)\left(1-b^{p}\right)^{2} \\
& \leq a^{\alpha(r-s t)+t-r} b^{\alpha(r+s p-s t)+t-r}(a-b)\left(a^{-t}-b^{p-t}\right)^{2}\left(a^{r-s t}-b^{r+s p-s t}\right)^{2}\left(a^{\alpha s p}-1\right) \\
& \quad \times\left(c_{11}+\frac{\alpha s p}{b} \frac{\delta}{\varepsilon}+\frac{o}{\varepsilon}\right) .
\end{aligned}
$$

We remark (2.3) implies

$$
\frac{1-b}{a-1+\varepsilon} \leq \frac{\delta}{\varepsilon}
$$

Hence, to get a contradiction, it seems reasonable to assume

$$
\delta=\frac{1-b}{a-1} \varepsilon
$$


Then, by letting $\varepsilon \rightarrow+0$, we have

$$
\begin{aligned}
& (a-1)\left(a^{-s t}-b^{s p-s t}\right)^{2}\left(1-b^{p}\right)^{2}\left(a^{\alpha(r+s p-s t)}-b^{\alpha(r+s p-s t)}\right)\left(a^{\alpha(r-s t)}-b^{\alpha(r+s p-s t)}\right) \\
& \leq a^{\alpha(r-s t)+t-r} b^{\alpha(r+s p-s t)-1+t-r}\left(a^{r-s t}-b^{r+s p-s t}\right)\left(a^{\alpha s p}-1\right) \\
& \times\left\{-\alpha s b\left(1-b^{p}\right)\left(a^{-t}-b^{-t}\right)\left(a^{-t}-b^{p-t}\right)\left(a^{r-s t}-b^{r+s p-s t}\right)(a-1)\right. \\
& \quad-\alpha a^{-t} b^{1-t}\left(1-b^{p}\right)^{2}\left(a^{r}-b^{s}\right)\left(a^{-s t}-b^{s p-s t}\right)(a-1) \\
& \left.+\alpha s p(1-b)(a-b)\left(a^{-t}-b^{p-t}\right)^{2}\left(a^{r-s t}-b^{r+s p-s t}\right)\right\} .
\end{aligned}
$$

Since (2.1) implies

$$
0<\alpha(r+s p-s t)-1+t-r
$$

we have, by letting $b \rightarrow+0$,

$$
0<(a-1) a^{-2 s t+\alpha(2 r+s p-2 s t)} \leq 0 .
$$

This is a contradiction.

\section{ACKNOWLEDGEMENTS}

The author would like to express his sincere thanks to the referee for heartwarming suggestions.

\section{REFERENCES}

[1] N. N. Chan and M. K. Kwong, Hermitian matrix inequalities and a conjecture, Amer. Math. Monthly, 92 (1985), 533-541. MR 87d:15011

[2] A. Aluthge, Some generalized theorems on p-hyponormal operators, Integr. Equat. Oper. Th., 24 (1994), 497-501. MR 97a:47032

[3] M. Fujii, T. Furuta, E. Kamei Complements to the Furuta inequality, Journal of the Japan Academy, 70 (1994), 239-242. MR 95j:47018

[4] T. Furuta, $A \geq B \geq O$ assures $\left(B^{r} A^{p} B^{r}\right)^{\frac{1}{q}} \geq B^{\frac{p+2 r}{q}}$ for $r \geq 0, p \geq 0, q \geq 1$ with $(1+2 r) q \geq(p+2 r)$, Proc. Amer. Math. Soc. 101 (1987), 85-88. MR 89b:47028

[5] T. Furuta, Applications of order preserving operator inequality, Oper. Theory Adv. Appl. 59 (1992), 180-190. MR 94m:47033

[6] T. Furuta, Furuta's inequality and its application to the relative operator entropy, J. Operator Theory, 30 (1993), 21-30. MR 95j:47019

[7] T. Furuta, Extension of the Furuta inequality and Ando-Hiai log-majorization, Linear Alg. and its Appl., 219 (1995), 139-155. MR 96k:47031

[8] T. Furuta, Generalized Aluthge transformation on p-hyponormal operators, Proc. Amer. Math. Soc., 124 (1996), 3071-3075. MR 96m:47041

[9] E. Heinz, Beiträge zur Störungstheorie der Spektralzerlegung, Math. Ann., 123 (1951), 415438. MR 13:471f

[10] K. Löwner, Über monotone Matrixfunktionen, Math. Z., 38 (1934), 177-216.

[11] K. Tanahashi, Best possibility of the Furuta inequality, Proc. Amer. Math. Soc. 124 (1996), 141-146. MR 96d:47025

[12] K. Tanahashi, The Furuta inequality with negative power, Proc. Amer. Math. Soc. (to appear). CMP 98:03

[13] T. Yoshino, A modified Heinz's inequality, (preprint)

Department of Mathematics, Tohoku College of Pharmacy, Komatsushima, Aoba-ku, SENDAI 981-8558, JAPAN

E-mail address: tanahasi@tohoku-pharm.ac.jp 